\title{
Astrophysics Titles in Scientific American Magazine (1990-2014): Linguistic and Discourse Practices
}

\author{
David I. Méndez (Corresponding author) \\ Department of Physics, System Engineering and Signal Theory, Polytechnic University College, University of Alicante, Carretera San Vicente del \\ Raspeig s/n - 03690 San Vicente del Raspeig - Alicante, Spain \\ E-mail: david.mendez@ua.es \\ M. Ángeles Alcaraz \\ Department of English Studies, Faculty of Arts, University of Alicante \\ Carretera San Vicente del Raspeig s/n - 03690 San Vicente del Raspeig - Alicante, Spain \\ E-mail: ariza@ua.es
}

Received: 18-03- 2015

Published: 01-11- 2015
Accepted: 20-06- 2015

doi:10.7575/aiac.ijalel.v.4n.6p.39
Advance Access Published: August 2015

URL: http://dx.doi.org/10.7575/aiac.ijalel.v.4n.6p.39

\begin{abstract}
We analyze Astrophysics titles published in Scientific American Magazine in the period 1990-2014 and compare them with Astrophysics titles of specialized journals. Our main results show that titles published in Scientific American are short, clear, direct and with low lexical density and little terminology. They mainly consist in simple and nominal constructions with few adjectives and compound groups. The predominance of nominal compounds and the high number of verbal titles and definite articles imply that popularized science titles mainly deal with global and wellestablished concepts. Pragmatic and rhetorical strategies are common in Astrophysics Scientific American titles in order to appeal to multiple audiences and invite them to use their cultural background knowledge to grasp at the actual meaning. Although pragmatic and rhetorical mechanisms overlap in some titles, rhetorical devices seem to prevail over pragmatic ones. All in all, however, both types of devices reveal a growing trend over time.
\end{abstract}

Keywords: Titles, Astrophysics, Scientific American Magazine, linguistic analysis, discourse practices

\section{Introduction}

Ever since humans appeared on Earth, stars have always stirred their fantasy due to their remoteness and the aura of mystery that surrounds them. What started first as a matter of magic, sorcery, astrology and their related superstitions led to the inception of Astronomy, a natural science which deals with the study of celestial objects. A few centuries later, Astronomy gave birth to Astrophysics, a discipline with a dual nature, i.e. a combination of Astronomy as an observational science related to the description and the classification of the universe, and of Physics, which is concerned with the basic properties of celestial bodies (Pedersen, 2009). Later on, the genuine scientific and technological knowledge developed by scientists in a restricted scientific community (Hilgartner, 1990) was provided to broad, interdisciplinary, scientific and non-scientific sectors of the population with different discursive practices (Jeanneret, 1994; Muñoz Dagua, 2010). As Sánchez Mora (2000) and Orrico (2012) claimed, popularization of science consists in building a bridge between the "world of science" and the "other worlds".

Scientific divulgation may be simply seen as reporting scientific facts to a non-specialist audience. However, the boundary between specialist and popularized discourses and between scientists and non-specialists is not as clear-cut as it might be thought because both types of discourse and communities interact in the process of knowledge construction (Myers, 2003; Fahnestock, 2004; Bucchi, 2008; among others). As Hyland (2010: 19) so rightly pointed out, "popular science does not just report scientific facts to a less specialist audience but represents phenomena in different ways to achieve different purposes". In this sense, scientific divulgation is a "civic" process that makes scientific issues understandable to general audiences (Kalleberg, 2000; Kyvik, 2005; Luzón Marco, 2013).

Whenever a given piece of specialized knowledge is communicated outside the domain where it was produced, it has to be adapted, re-contextualized, re-constructed or re-formulated (Jacobi, 1987; Villaroya, 2013) in such a way that potential receivers are able to interpret and integrate it into their existing knowledge (Crismore, \& Farnsworth, 1990; Alcíbar Cuello, 2004; Calsamiglia, \& Van Dijk, 2004; Ciaspuscio, 2005; Cortiñas, 2008; Rodríguez Estrada, \& Davis, 2015). Apart from choosing the appropriate words to explain complex concepts, a very important element that scientific divulgators have to take into consideration is the contextual dimension: what they want to communicate, to whom, what they intend to achieve (Frankel, \& DePace, 2012), the pre-existing knowledge of the world they share with their audience (Jay, 2002), and the channel through which they want to transmit their message.

Popularized science communicators may include different disciplinary communities with their theoretical and methodological approaches. They are usually researchers, professional science journalists, and amateur astronomers in the case of Astrophysics. Popularized science audience is formed by multiple publics with shared interests, including 
experts both inside and outside the given research field with different degrees of expertise, and non-specialist audiences although with some training in science. In this sense, there is not a single boundary between scientists and non-scientists but a multitude of partnerships at many different levels (Lévy-Leblond, 2001; Cartellier, 2010). As Myers (1990) accurately put it, two conceptions of science are thus merging: 1) a narrative of nature proper of scientific divulgation; and 2) a narrative of science proper of scientific research. Moreover, only those scientific facts likely to be of interest to the target public outside the field of expertise are usually communicated. Conversely to scientific knowledge, which is transmitted by specialized books and/or journals and conference presentations, popularized science is more broad ranging and is presented in many forms that include film, television and radio documentaries, internet, magazine articles, museums and/or exhibitions, etc.

A very effective discursive strategy used to capture the attention of potential readers and engage them in what is being communicated, both in scientific and popularized science texts, is the use of adequate titles. Indeed, titles play a double capital role since, as Swales (1990: 179) so rightfully claimed, they are both a "front and summary matter'. In the academic community, titles allow academics decide on the relevance and usefulness of a given paper to their own area of interest. To this end, they have to be clear, accurate and precise. As a matter of fact, the more precise and accurate a title is, the easier it will attract the attention of and inform its primary target audience, editors and reviewers, and the easier it will be for bibliographers to compile data for indexing, abstracting and other documentation purposes. Conversely, popularized science titles may be said to belong to the industry of advertising and journalism, which means that they have primarily to be eye-catching, creative, innovative, and even deliberately ambiguous, in order to appeal to their possible audience and arouse their interest.

Due to their importance in academic writing, research on scientific titles has been approached in a wide range of academic contexts and from different perspectives (cross-generic, cross-disciplinary, cross-linguistic, monodisciplinary, etc. $)^{\mathrm{i}}$. Among the numerous studies that have examined title wording, we can mention those by Fortanet, Posteguillo, Coll, \& Palmer (1998), Anthony (2001), Wang, \& Bai (2007), or Cheng, Kuo, \& Kuo (2012). Fortanet, Posteguillo, Coll, \& Palmer (1998) observed the prevalence of the definite article in research paper titles in Applied Linguistics, Business and Economy. Anthony (2001), who studied the length, word frequency, prepositions, and punctuation marks in the RP titles of various Computer Science sub-disciplines, discovered that there were statistically significant differences in the most common words from journal to journal, showing the sub-disciplinary content specificity. Wang, \& Bai (2007) also revealed that full sentence, prepositional and -ing phrase titles in medical RPs occurred much less frequently than compound or nominal structures. In a similar vein, Cheng, Kuo, \& Kuo (2012) noted the prevalence of compound and nominal titles, as well as of -ing and prepositional phrases in RPs published in Applied Linguistics.

Summing up, all the studies that have dealt with these "texts in miniature" (Haggan, 2004: 20) have shown that they vary and at the same time maintain a series of similarities across a number of factors and in several dimensions including functions, informativity, length, punctuation marks, structure, or wording. There is however a discipline where titles have been addressed on very few occasions. We are referring to Astrophysics, a multidisciplinary science that combines separate branches of fields of expertise, each one with its own characteristics (Particle Physics, Relativistic Physics, Solid-state Physics, Mathematics, Chemistry, Biology, Mechanical/Electrical/Aerospace Engineering, etc.). Our purpose in this paper is thus to complete our previous research on academic titles in Astrophysics (Méndez, Alcaraz, \& Salager-Meyer, 2014; Méndez, \& Alcaraz, 2015) with an analysis of popularized titles published in the same field.

\section{Corpus \& purpose}

Since science popularization serves to bridge the gap between the world of science and society at large, we drew our corpus of titles from Scientific American Magazine (from now on abbreviated as SciAm). SciAm journal is one of the most internationally prestigious journals in the divulgation of science where scientists and professional science journalists from diverse fields present studies to a very wide audience formed by both scientists that search information about areas outside their expertise and the general public interested in the issues discussed. We considered that a period of 25 years would allow us to reach some suitable results and this is why we compiled all the titles related to astrophysical matters published between 1990 and 2014 from the printed version of the journal.

If science popularization means science re-contextualization, it is no doubt that the transfer of knowledge from a specialized context to a less specified will involve some changes at different levels. Therefore, our study aims at answering the following questions:

(1) Do popularized science titles differ from specialized titles in terms of their linguistic formulation, and if they do, in what way and to what extent?

(2) Which are the specific devices, if any, used when transmitting the information created in a given specialized context to a more general one?

(3) Has the distribution of the referred specific devices, if any, changed over time?

To answer question (1), we carried out a comparative analysis between titles of papers dealing with astrophysical matters published in SciAm and titles of research papers published in the most prestigious journals in Astrophysics (see Méndez, Alcaraz, \& Salager-Meyer, 2014; Méndez, \& Alcaraz, 2015). To answer question (2), we systematically looked for the different discursive practices used by scientific divulgators to engage their diverse audiences with scientific issues. To answer question (3), we examined the evolution of the traced discursive practices to observe their variations. 


\section{Methodology}

To find out the linguistic differences between titles published in SciAm and titles published in specialized journals, we analysed the following linguistic variables: title length, title lexical density, and title types.

\subsection{Title length (counted as the number of running words)}

We manually counted all the words included in the titles. The concept of "word" was defined as the unit occurring between spaces. Each word making up capitalized abbreviations was counted as one word: for instance, 'SNU' $<$ Solar Neutrino Unit) was counted as three different words. Acronyms ${ }^{\mathrm{ii}}$ were counted as one word: for example, 'quasar' $(<$ QUAsi-StellAR radio source) was counted as one word. Hyphenated words were counted according to the number of their semantic components: 'gamma-ray', for instance, was counted as two words (see also Méndez, \& Alcaraz, 2015).

\subsection{Title lexical density}

In order to determine title lexical density, i.e. the amount of information conveyed by titles, we divided the words found in our corpus into lexical or content words (nouns, adjectives, adverbs, past and present participles, conjugated and infinitive verbs) and grammatical or function words (auxiliary verbs, determiners -definite and indefinite articles, possessives-, conjunctions, prepositions, pronouns, and wh-words). Here-below are some examples that illustrate content and function words in both types of titles (the words corresponding to each specific linguistic variable are underlined):

\subsubsection{Lexical/content words}

(1) Collisions with Comets and Asteroids (SciAm - February 1996)

(2) The globular cluster systems of the early-type galaxies NGC 3379, NGC 4406, and NGC 4594 and implications for galaxy formation (The Astronomical Journal - January 2004)

\subsubsection{Grammatical/functions words}

(3) The Flip Side of the Universe (SciAm - September 1998)

(4) The lower main sequence of the globular cluster M3 with the Hubble Space Telescope: luminosity and mass functions (Monthly Notices of the Royal Astronomical Society - February 1998)

\subsection{Title types}

Titles may be classified into simple or compound. Simple titles consist of a general heading, whereas compound titles comprise a general heading followed by a specific theme generally separated by a punctuation mark. Another title type distinction, which is non-excluding with the previous one, may also be established, and it is the one that differentiates nominal from verbal titles. A nominal title does not contain any conjugated verb; on the contrary, a verbal title contains an active verb with a full sentence that usually states the findings or the conclusion of the research being reported ${ }^{\text {iii }}$. Furthermore, simple, compound, nominal or verbal constructions may also be formulated as questions. Here-below are some examples that illustrate the different title types in both corpora.

3.3.1 Simple/nominal titles

(5) Planetary Protection (SciAm - October 2002)

(6) Evolutionary and pulsational properties of low-mass white dwarf stars with oxygen cores resulting from close binary evolution (Monthly Notices of the Royal Astronomical Society - January 2004)

3.3.2 Compound/nominal titles

(7) Mercury: The Forgotten Planet (SciAm - November 1997)

(8) Infrared array photometry of bulge globular clusters

I. Combined ground based JK and HST VI photometry of NGC 6553 (Astronomy and Astrophysics - March 1998)

\subsubsection{Verbal titles}

(9) You Are Here (SciAm - November 2012)

(10) The CHARA array angular diameter of 8799 favors planetary masses for its imaged companions (The Astrophysical Journal - December 2012)

3.3.4 Nominal/verbal/question titles

(11) What is Happening at the Center of our Galaxy? (SciAm - April 1990)

(12) Where Are They? (SciAm - July 2000)

(13) Constraining the population of cosmic ray protons in cooling flow clusters with X-ray and radio observations: Are radio mini-halos of hadronic origin (Astronomy \& Astrophysics - January 2004)

\section{Results}

\subsection{Linguistic analysis}

The information about the linguistic variables analysed (number of titles, their length, the word average per title, and the number of content and function words) for both corpora is shown in Table 1. 
Table 1. Linguistic variables

\begin{tabular}{ccc}
\hline Variables & SciAm & Specialized journals \\
\hline N. ${ }^{\circ}$ of titles & 329 & 300 \\
\hline N. $^{\circ}$ of words & 1404 & 4047 \\
\hline Word average & 4.27 & 13.49 \\
\hline No. ${ }^{\circ}$ of content words & $950(67.7 \%)$ & $3088(76.3 \%)$ \\
\hline N. $^{\circ}$ of function words & $454(32.3 \%)$ & $959(23.7 \%)$ \\
\hline
\end{tabular}

As it can be seen in Table 1, title word average in popularized science titles is more than three times lower than in scientific ones. Table 1 also shows that although content words outnumber function words in both corpora, the percentage of content words as compared to the number of function words is also lower in popularized science titles than in scientific ones.

Table 2 displays the breakdown of the different types of content and function words in both types of titles.

Table 2. Breakdown of content and functions words

\begin{tabular}{ccc}
\hline Content Words & SciAm & Specialized journals \\
\hline Nouns & $607(63.90 \%)$ & $1873(60.65 \%)$ \\
\hline Qualifying adjectives & $221(23.26 \%)$ & $973(31.51 \%)$ \\
\hline Verbs & $35(3.68 \%)$ & $10(0.32 \%)$ \\
\hline -ing verbs & $32(3.37 \%)$ & $45(1.46 \%)$ \\
\hline Adverbs & $28(2.95 \%)$ & $25(0.81 \%)$ \\
\hline ed-adjectives & $14(1.47 \%)$ & $66(2.14 \%)$ \\
\hline -ing adjectives & $8(0.84 \%)$ & $37(1.20 \%)$ \\
\hline -ing nouns & $5(0.53 \%)$ & $25(0.81 \%)$ \\
\hline Symbols & $0(0 \%)$ & $34(1.10 \%)$ \\
\hline Function Words & $\boldsymbol{S c i A m}$ & $229(23.88 \%)$ \\
\hline Definite articles & $179(39.43 \%)$ & $517(53.91 \%)$ \\
\hline Prepositions & $176(38.77 \%)$ & $83(8.65 \%)$ \\
\hline Indefinite articles & $42(9.25 \%)$ & $121(12.62 \%)$ \\
\hline Conjunctions & $20(4.41 \%)$ & $3(0.31 \%)$ \\
\hline Wh-words & $14(3.08 \%)$ & $1(0.10 \%)$ \\
\hline Pronouns & $9(1.98 \%)$ & $3(0.31 \%)$ \\
\hline Auxiliary verbs & $8(1.76 \%)$ & $2(0.21 \%)$ \\
\hline Possessives & $6(1.32 \%)$ & Specized journals \\
\hline
\end{tabular}

The percentage of nouns, verbs, -ing verbs and adverbs is higher in popularized science titles than in scientific ones, especially that of verbs which amounts 11.5 times as much in the former than in the latter. Conversely, the percentage of qualifying adjectives, -ing nouns, -ing- and -ed adjectives is slightly lower in popularized science titles. It is interesting to note that the Saxon genitive has been registered on 12 occasions in popularized science titles and that of all the qualifying adjectives recorded in the titles published in SciAm, seven (3.2\%) are formulated in comparative form and five $(2.7 \%)$ in superlative form. On the contrary, in specialized journals comparative and superlative adjectives only amount to $0.5 \%$ and $0.2 \%$, respectively. Also worth adding is that no symbol, either chemical or mathematical, was registered in the titles drawn from SciAm.

As for functions words, the percentage of auxiliary verbs, definite articles and possessives is much higher in SciAm, especially that of auxiliary verbs and possessives which amounts more than five and six times respectively in popularized science titles than in scientific ones. The higher percentage of pronouns in popularized science titles is worth noting since only one pronoun was recorded in scientific titles. The percentage of wh-words is also much higher, about nearly ten times as much as in specialized titles. The percentages of indefinite articles are similar in both types of titles, whereas the percentage of prepositions and conjunctions is much lower in popularized science titles.

A further lexical distinction in the category of content words is shown in Table 3. 
Table 3. Other lexical features (percentages in relation to the total number of words)

\begin{tabular}{ccc}
\hline Variables & SciAm & Specialized journals \\
\hline Proper names & $55(30.26 \%)$ & $43(1.06 \%)$ \\
\hline Foreign words & $51(3.63 \%)$ & $117(2.89 \%)$ \\
\hline Eponyms & $10(0.71 \%)$ & $122(3.01 \%)$ \\
\hline Acronyms & $9(0.64 \%)$ & $43(1.06 \%)$ \\
\hline Abbreviations & $6(0.43 \%)$ & $366(9.04 \%)$ \\
\hline Numbers & $5(0.36 \%)$ & $225(5.56 \%)$ \\
\hline Toponyms & $1(0.07 \%)$ & $10(0.25 \%)$ \\
\hline Compound nouns & $57(73.1 \%)$ & $310(64.6 \%)$ \\
\hline Compound adjectives & $21(26.9 \%)$ &
\end{tabular}

The percentage of proper names, which refer mainly to stars, planets, satellites and galaxies, is much higher in popularized science titles. By contrast, the percentage of eponyms (proper names) and toponyms (names of places) applied to astronomical devices, acronyms, abbreviations and numbers is much lower in popularized science titles. In foreign words (Greek, Latin and other languages), there is not much difference between the percentages in both types of titles.

On many occasions, both popularized and scientific Astrophysics titles also contain compound groups (nominal and/or adjectival ones). Compound groups are compressed structures where information is condensed through the juxtaposition of content words without any function word, as is shown in the following titles:

(14) Crater Jumper (SciAm - March 2006) (compound noun)

(15) Black Hole Blowback (SciAm - March 2007) (compound adjective)

(16) Jet-induced shocks in 3c 171: an intermediate-redshift analog of high-redshift radio galaxies (The Astrophysical Journal - February 1998) (compound noun and compound adjectives)

Two interesting points are worth noting in relation to compound groups: 1) the compound group average is much lower in popularized science titles $(0.24$ per title) than in specialized titles (1.6 per title), i.e. its amounts six times as much in the latter as in the former; 2) the percentage of compound nouns is higher in popularized science titles, whereas the percentage of compound adjectives is higher in specialized titles.

Table 4 shows that titles published in SciAm include more simple structures and much less compound ones than those published in specialized journals. The occurrence of nominal constructions does not differ much in both types of titles. Verbal and question structures are more frequent in popularized science titles. With respect to exclamatory titles, they are only present in popularized science titles although in a very small percentage as only two occurrences were recorded. Here-below is one of the two exclamatory titles, which also contains two discourse devices. On the one hand, the pragmatic allusion to 'Jove', another name for Jupiter, the largest and most massive planet in the solar system, refers to Roman mythology and, on the other hand, it is a clear example of "ecphonesis", a rhetorical strategy used to express strong emotion and surprise (see sub-sections 4.1. and 4.2).

(17) By Jove! (SciAm - October 1994)

Table 4. Titles types

\begin{tabular}{ccc}
\hline Title type & $\boldsymbol{S c i A m}$ & Specialized journals \\
\hline Simple & $322(97.87 \%)$ & $195(65 \%)$ \\
\hline Compound & $7(2.13 \%)$ & $105(35 \%)$ \\
\hline Nominal & $295(89.67 \%)$ & $291(97 \%)$ \\
\hline Verbal & $34(10.33 \%)$ & $9(3 \%)$ \\
\hline Question & $17(5.17 \%)$ & $11(3.67 \%)$ \\
\hline Exclamatory & $2(0.61 \%)$ & $0(0 \%)$ \\
\hline
\end{tabular}

According to Table 5, the punctuation marks mostly used in popularized science compound constructions are the dash and the colon. Conversely, specialized compound titles recur mostly to the colon, the full stop and two-lines. 
Table 5. Punctuation marks

\begin{tabular}{ccc}
\hline Punctuation marks & SciAm & Specialized journals \\
\hline Dash & $11(44 \%)$ & $4(3.33 \%)$ \\
\hline Colon & $7(28 \%)$ & $63(52.50 \%)$ \\
\hline Comma & $6(24 \%)$ & $11(9.17 \%)$ \\
\hline Semi-colon & $1(4 \%)$ & $0(0 \%)$ \\
\hline Full Stop & $0(0 \%)$ & $27(22.50 \%)$ \\
\hline Two-lines & $0(0 \%)$ & $15(12.50 \%)$ \\
\hline
\end{tabular}

It is worth adding that contrary to popularized science titles, the commas that were found in specialized titles did not mark any boundary between simple and compound structures because they were not used to specify things but to enumerate them.

(18) Bright Lights, Big Mystery (SciAm - August 1998)

(19) An attempt to probe the radio jet collimation regions in NGC 4278, NGC 4374 (M84), and NGC 6166 (The Astronomical Journal - January 2004)

Apart from the comma which is used to specify two different concepts, Title (18) contains the figure of speech known as 'asyndeton' (see sub-section 4.2.), which consists in grouping together two opposite clauses.

A further difference worth noting between both types of titles is related to typographical matters. All the words of the titles published in SciAm are written with initial capital letters, except prepositions. By contrast, in titles published in specialized journals, only the initial word in simple titles and on some occasions the word following the punctuation marks in compound titles appear with capital letters.

\subsection{Discourse analysis}

Pragmatic and rhetorical strategies are practically non-existent in titles published in specialized journals. By contrast, authors of popularized science papers use a variety of pragmatic and rhetorical devices in their titles in order to appeal to their intended audience (Leech, 1983; Horn, \& Ward, 2004; Kecskes, \& Horn 2007). Although both types of strategies may overlap in some titles, for the sake of a clearest analysis we have opted to deal with them separately.

\subsubsection{Pragmatic strategies}

As can be inferred from Table 6, the only pragmatic strategy that has been traced in our corpus of science popularized titles consists of references to different referents. We have found a total of 134 references, which we have classified into 13 different categories, five titles containing two references each. In other words, more than two fifths (40.7\%) of all the titles contains a least one reference.

Table 6. Pragmatic strategies

\begin{tabular}{cc}
\hline Pragmatic devices & Ocurrences \\
\hline References to journalistic discourse & $54(40.4 \%)$ \\
\hline References to literary discourse & $21(15.7 \%)$ \\
\hline References to movies & $17(12.7 \%)$ \\
\hline References to books and comics & $13(9.7 \%)$ \\
\hline References to pop music & $10(7.5 \%)$ \\
\hline References to TV series & $5(3.7 \%)$ \\
\hline References to foreign cultures & $3(2.2 \%)$ \\
\hline References to tales, myths and legends & $3(2.2 \%)$ \\
\hline References to History & $2(1.5 \%)$ \\
\hline References to sports & $2(1.5 \%)$ \\
\hline References to videogames & $2(1.5 \%)$ \\
\hline References to Economics & $1(0.7 \%)$ \\
\hline References to weather forecast & $1(0.7 \%)$ \\
\hline Total & $134(100 \%)$ \\
\hline
\end{tabular}

References to journalistic discourse top the frequency scale of the referent categories and usually include conversational elements such as contractions, idioms and phrasal verbs:

(20) What's The Matter? (SciAm - May 2000)

(21) The Cosmic Reality Check (SciAm - March 2002) 
(22) How to Blow Up a Star (SciAm - October 2006)

The poetic function proper of literary discourse is another helpful device to popularize scientific information:

(23) Whispers from Creation (SciAm - August 2014)

References to famous movies, books, pop music and TV series are often exploited to create an atmosphere of intimacy and proximity:

(24) Making a Deep Impact (SciAm - May 1998) (referring to the movie "Deep Impact", directed by Mimi Leder and whose premiere took place exactly on May 8, 1998)

Some years later, exactly in May 2001, the reference to the same movie, although formulated in a comparative form, appears in the following title:

(25) Deeper Impact (SciAm - May 2001)

(26) A Hundred Billion Years of Solitude (SciAm - April 1999) (referring to Gabriel García Márquez’s book “One hundred years of solitude")

(27) Here Come the Suns (SciAm - May 1999) (referring to the famous Beatles' song "Here comes the sun" written by George Harrison)

(28) The Biggest Bang Theory (SciAm - October 2010) (referring to the well-known sitcom "The Big Bang Theory" whose fourth season premiered on CBS, exactly on September 23, 2010)

The remaining references cluster around different frequencies, the least frequent ones being related to the discourse of Economics and of weather forecast:

(29) Goldilocks Black Holes (SciAm - January 2012) (referring to a little girl in the classic children's story "The Three Bears") $)^{\text {iv }}$

(30) Origami Observatory (SciAm - October 2010) (referring to the Japanese art of paper folding)

(31) Inflation is Dead; Long Live Inflation (SciAm - July 1998) (referring to the crowning of a new king, a traditional proclamation in various countries)

It is worth noting that Title (31) includes the only semi-colon that was registered in our corpus of popularized science titles.

Some titles may also refer to other topics:

(32) Moonball (SciAm - July 1993) (referring to tennis terminology)

(33) Star Gobbler (SciAm - August 1994) (referring to "Gobbler", an Apple clone of the well-known Pac-Man arcade game)

(34) Boom or Bust (SciAm - October 1999) (referring to the "boom and bust" economic cycles)

(35) Cloudy with a Chance of Stars (SciAm - February 2010) (referring to the typical expression of weather forecast "Cloudy with a chance of rain")

\subsubsection{Rhetorical strategies}

As can be seen in Table 7, a total of 199 rhetorical devices with 26 variants (see Dupriez, 1991 for a comprehensive list of rhetorical devices) were found in 169 titles, i.e. more than half of the titles $(51.4 \%)$ contain rhetorical mechanisms. Furthermore, 28 titles include two rhetorical devices $(8.5 \%)$ and one title even includes three rhetorical resources $(0.3 \%)$.

Table 7. Rhetorical strategies

\begin{tabular}{cc}
\hline Rhetorical devices & Occurrences \\
\hline Prosopopoeia & $59(29.6 \%)$ \\
\hline Metaphor & $47(23.6 \%)$ \\
\hline Synecdoche & $26(13.1 \%)$ \\
\hline Double entendre & $20(10.1 \%)$ \\
\hline Circumlocution & $6(3.1 \%)$ \\
\hline Antithesis & $5(2.5 \%)$ \\
\hline Ellipsis, epithet & $4(4$ each $)(4 \%)$ \\
\hline Hyperbaton, hyperbole, paradox, paronomasia & $9(3$ each) $(4.5 \%)$ \\
\hline $\begin{array}{c}\text { Alliteration, apostrophe, asyndeton, chiasmus, } \\
\text { ecphonesis, emphasis, metononymy, } \\
\text { onomomatopoeia, parallelism, pleonasm, } \\
\text { reduplication }\end{array}$ & $8(2$ each) $(4 \%)$ \\
\hline Total & $11(1$ each $)(5.5 \%)$ \\
\hline
\end{tabular}


By far the most frequent rhetorical strategies to tailor information to the readers are prosopopopeias, metaphors, synecdoches and double entendres. Prosopopoeias alone account for the $29.6 \%$ of all the rhetorical resources. This rhetorical device is used to personify objects by attributing them human characteristics which on some occasions are associated with the use of the Saxon genitive, like in the following example:

(36) Through Titan's Haze (SciAm - January 2005)

Like prosopopoeias, metaphors and synecdoches help to reconceptualize new areas of knowledge into more familiar ones. For instance, the metaphor in title (30) clearly compares stellar vibration to bells' vibration. In addition, this title also includes a pragmatic device as it makes a direct reference to Tubular Bells, the Mike Oldfield's debut album released in 1973.

(37) Stellar Bells (SciAm - November 1992)

A title with a double synecdoche is the following one, where the wide concept "worlds" substitutes for the restricted concept "planets". Conversely, the specific word "suns" substitutes for the more general concept "stars":

(38) Worlds with Two Suns (SciAm - November 2013)

It is worth mentioning that all the double entendres found in our corpus have no indelicate meanings. A striking example of this figure of speech is the following title, where its author plays with the word "wave". Three complementary meanings may be said at stake: the literal meaning of a surf expression, the figurative meaning of understanding and behaving according to the most modern fashions or using the most innovative techniques and, finally, the actual meaning related to the possible detection of gravitational waves:

(39) Catching the Wave (SciAm - March 1992)

The presence of circumlocution, antithesis, ellipsis, epithet, litotes and oxymoron ${ }^{\mathrm{v}}$ is also worth mentioning. Circumlocution is proper of poetry and it consists in using more words to name a concept instead of naming it directly as in the case of the following title, where Pluto is referred to as "the farthest planet":

(40) Journey to the Farthest Planet (SciAm - May 2002)

When two words are introduced in close proximity to one another for contrasting effect, we have an antithesis:

(41) From Slowdown to Speedup (SciAm - February 2004)

Ellipsis, which has to do with the omission of some parts of a sentence, is proper of the following title:

(42) The Little Spacecraft that Could (SciAm - June 1999)

Title (42) also refers to "The Little Engine that Could", an illustrated story that was published in the United States in 1930 the aim of which was to teach children the values of optimism and hard work.

Epithet, i.e. an adjective used to emphasize the intrinsic characteristics of something, is illustrated in Title (43):

(43) New Beginnings (SciAm - October 2007)

Apart from being typical of the language of journalism, Title (44) includes three different rhetorical mechanisms:

(44) COBE Corroborated (SciAm - February 1993)

a) Metonymy: the arbitrarily constructed acronym "COBE" (< COsmic Background Explorer) stands for the results obtained by the referred satellite;

b) Hyperbaton: the order of the words does not follow the English usual one of adjective + noun;

c) Cacophony: the syllable "CO" appears at the beginning of two consecutive words.

The figures of speech known as 'litotes', which consists in stating a negative to further affirm a positive, and 'oxymoron', which juxtaposes apparently contradictory elements, are illustrated in the following titles:

(45) The Not-So-Dark Matter (SciAm - April 2007)

(46) Inconstant Constants (SciAm - November 1998)

Finally, we divided the period of our study (1990-2014) in sections of five years to check if the frequency of occurrence of the traced discourse mechanisms has changed over time. The evolution of discourse mechanisms per title is displayed in Graph 1. 


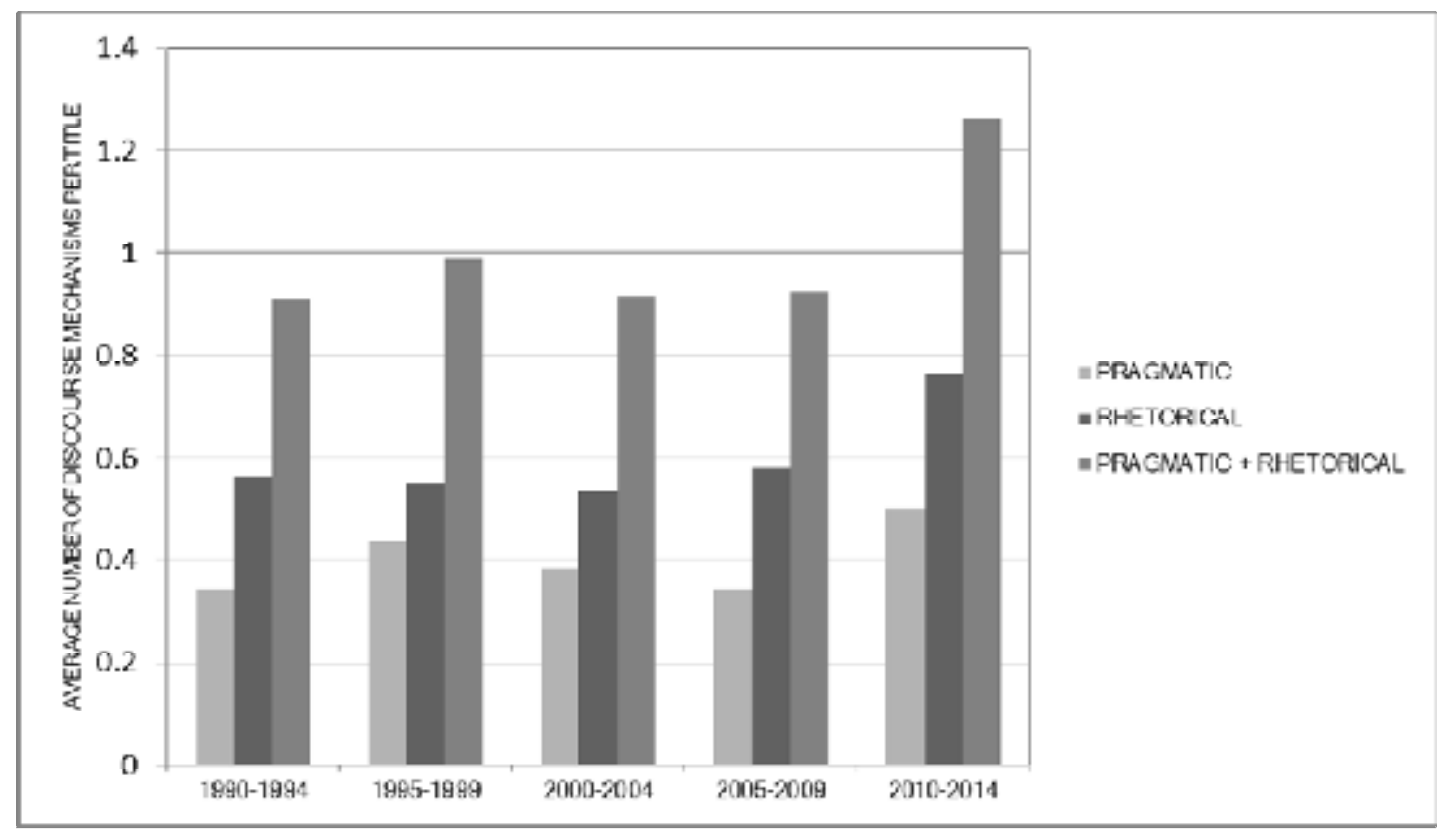

Figure 1. Evolution of pragmatic and rhetorical devices

As it can be seen in Graph 1, rhetorical devices prevail over pragmatic ones. Moreover, rhetorical mechanisms show a more or less stable distribution from 1990 to 2014, whereas pragmatic resources show an erratic up-down-up pattern over time. All in all, however, both types of devices and their variants tend to grow between the initial and last periods.

\section{Discussion}

As our linguistic analysis has put forward, titles related to astrophysical matters published in SciAm are easier to understand by a general public than those published in specialized Astrophysics journals, which are specifically addressed to a more restricted audience. Titles in SciAm are short and formulated in a clear and direct way, with no syntactic complexity and with less semantic richness and specificity than those published in specialized journals. They consist primarily in simple and nominal constructions with few adjectives. Likewise, the low presence of compound groups and of terminology related to astrophysical matters (numbers, symbols, abbreviations, acronyms, and toponyms) implies that the titles of papers published in SciAm do not belong to specialized excerpts. However, the use of foreign words (Greek, Latin and other languages) and references to history, legend, literature, myths and tales implies that this journal is addressed to an audience with educated level.

Likewise, the predominance of compound nouns over compound adjectives and the use of proper names, which refer mainly to the stars, planets, satellites and galaxies that possible readers likely know, imply that popularized science titles mainly deal with more global and established concepts. The higher number of verbal titles and of definite articles in popularized science papers when compared to specialized ones may also be interpreted as a desire to generalize the ideas presented. In this sense, popularized science titles somehow differ from scientific titles which are hardly formulated in verbal form because cutting-edge findings in Astrophysics tend always to be approximate. This comes as no surprise since the discipline mainly deals with distant objects of which only images or spectra can be obtained. Consequently, Astrophysics is not a science that strictly follows the usual "scientific method" of testing, hypothesis and refutation like, for example, Biology or Chemistry, where essays in laboratories allow investigators to repeat and modify the experimental conditions in order to obtain more reliable and trustworthy results.

Another strategy that popularized science writers use to appeal to potential audience and engage them in reading the papers is the use of question titles. Question titles arouse readers' curiosity by inviting them to find an answer to the question asked in the title (Calsamiglia, \& Van Dijk, 2004; Fahnestock, 2004; Giannoni, 2008; Hyland, 2010; Maisonneuve, Lorette, Maruani, \& Huguier, 2010; Goodman, 2011; Soler, 2011; or Luzón Marco, 2013 to name just a few), either by searching it in their own cultural background or by exploring it elsewhere, for instance in Wikipedia or other links. In this sense, question titles may be said to directly involve the addressees of the message in a sort of dialogue that contributes to the expansion of their general knowledge. Moreover, question titles in science popularized texts seem to stand in clear opposition to academic texts, where they are not usually favoured (Day, 1998; Busch-Lauer, 2000; Lewison, \& Hartley, 2005; Hartley, 2007; Soler, 2007; Salager-Meyer, Alcaraz Ariza, \& Luzardo Briceño, 2013, among others).

In the same vein as question titles, and also proper of journalistic and advertising styles, conversational elements such as contractions, idioms and phrasal verbs play an essential role in creating a persuasive message. Adjectives, formulated in comparative and superlative forms, as well as exclamatory phrases and sentences, are used to express emotional attitudes and strong feelings like excitement or surprise. Capitalized initial letters are also used to reflect emphasis. Personifying some objects, among others through the use of the Saxon genitive, relates these items to human beings 
with their emotions and innate curiosity. The dialogic involvement of addressing readers directly by means of personal pronouns and possessives enables to establish a closer relationship between senders and receivers. Involving readers in the dialogic process is moreover reinforced by the simultaneous use of pragmatic and rhetorical devices. Apart from adding interest and vividness to titles in order to make them very eye-catching, these strategies undoubtedly contribute to proximity by inviting potential readers to use their cultural knowledge and imagination to grasp at the actual meaning.

In this sense, and despite the potentially educated audience of SciAm, it is quite understandable that rhetorical devices are more frequent than pragmatic ones since the latter always require a higher dose of cooperation and a more specific readers' background. Within the repertoire of rhetorical devices, it comes as no surprise that prosopopoeias, metaphors and synecdoches are highly used as they are some of the most characteristic and striking figures of speech. Prosopopoeias, which are used to personify objects and thus have very much to do with human beings, undoubetdly make concepts easier to transmit. Likewise, metaphors and synecdoches are easily understandable because general knowledge is only needed to grasp at their meaning. Contrary to prosopopoeias, metaphors and synecdoches, double entendres wink at more educated readers and require not only their active participation but also their complicity for disambiguating their meanings.

As already stated, the educated audience of SciAm also accounts for the high number of references to different referents because, without a shadow of a doubt, in the scenario of written titling practices they are the best and easiest way to attract the largest possible audience and convince them to read the whole article. This is also the reason why the references most frequently used have to do with the language of journalism which is easily understandable since almost everyone reads journals occasionally. Conversely to journalistic discourse, references to literary discourse target at a more learned audience, hence their comparatively lower frequency of occurrence. Moreover, as in the case of journalistic discourse, references to movies, books, comics, pop music and TV series point to a wider audience that is abreast of what happens around them.

As for the overall increase over time of the number of pragmatic and rhetorical devices found in the titles published in SciAm, it has to be taken into account that a better understanding of science is important for everyone. Since most scientific research is publicly funded, scientists need to justify the funds they have been granted, especially in times of economic crisis, by reporting the results of their research so that the value of their work is understood and accepted by society at large, academics outside their community, intellectuals who discuss new scientific research publicly in order to "influence political, economic, social or cultural issues" (Kyvik, 2005: 290), and lay audience. The new role of "civic scientist" assumed by scientists is reflected in the changing trend of global knowledge society where any information is affordable to everyone. As Rodríguez Estrada, \& Davis (2015: 144) so rightly stated, "Only by seeing the world through its audience's eyes can science communicators build durable and beneficial audience relationships."

\section{Conclusions}

In this paper, we have conducted research on the linguistic and discourse practices of all the Astrophysics titles published in Scientific American Magazine, an internationally prestigious journal in the divulgation of science, over the past quarter century (1990-2014). More specifically, unlike previous studies (see Introduction section) our analysis has also included a systematic search of all the pragmatic and rhetorical strategies present in our corpus. Likewise, we have carried out a linguistic comparative analysis between the referred titles and those of research papers published in the most prestigious journals in Astrophysics (see Méndez, Alcaraz, \& Salager-Meyer, 2014).

The material presented here supports the following general conclusions:

1) Astrophysics SciAm titles are on average more than three times shorter than titles of research papers published in the most prestigious journals in Astrophysics.

2) Lexical density is lower in popularized science titles than in scientific ones.

3) Astrophysics SciAm titles are always formulated in a clear and direct way, with no syntactic complexity since they consist primarily in simple and nominal constructions with a low presence of adjectives, compound groups and specific terminology.

4) The predominance of nominal compounds over adjectival ones and the use of proper names, which refer mainly to the stars, planets, satellites and galaxies that readers probably know, imply that popularized science titles mainly deal with more global and established concepts.

5) The higher number of verbal titles and of definite articles in popularized science titles when compared to specialized titles may also be interpreted as a desire to generalize the ideas presented.

6) Apart from adding interest and vividness, the use of pragmatic and rhetorical strategies in Astrophysics SciAm titles appeals to multiple audiences and invites them to use their cultural background knowledge and imagination to discover the meaning of the information conveyed.

7) Pragmatic resources only consist of references to different referents. References to journalistic and literary discourses top the frequency scale in Astrophysics SciAm titles.

8) Figures of speech such as prosopopoeias, metaphors, synecdoches and double entendres with no indelicate meanings are the rhetorical devices most frequently used.

9) Although pragmatic and rhetorical mechanisms overlap and go hand in hand in some Astrophysical SciAm titles, 
rhetorical devices seem to prevail over pragmatic ones.

10) Pragmatic and rhetorical devices in Astrophysics SciAm titles reveal a growing trend over time.

\section{Acknowledgements}

We are grateful to Mercedes Blanes Jover for her valuable help in the analysis and classification of the different pragmatic and rhetorical devices found in the corpus studied.

\section{References}

Alcíbar Cuello, M. (2004). La divulgación mediática de la ciencia y la tecnología como recontextualización discursiva. Análisi: Quaderns de comunicació i cultura, 31, 43-70.

Anthony, L. (2001). Characteristic features of research article titles in computer science. IEEE Transactions of Professional Communication, 44(3), 187-194. http://dx.doi.org/10.1109/47.946464.

Bucchi, M. (2008). Of deficits, deviations and dialogues: Theories of public communication of science. In Massimiano Bucchi, \& Brian Trench (Eds.), Handbook of Public Communication of Science and Technology, 57-76. London: Routledge.

Busch-Lauer, Ines-A. (2000). Titles in English and German research papers in medicine and linguistics. In Anna Trosborg (Ed.), Analysing Professional Genres, 77-97. Amsterdam: John Benjamins.

Calsamiglia, H., \& Van Dijk, T. (2004). Popularization discourse and knowledge about the genoma. Discourse in Society, 15, 369-389. http://dx.doi.org/10.1177/0957926504043705.

Cartellier, D. (2010). La vulgarisation scientifique à l'heure de libre accessibilité des savoirs. Quelle place pour les médiateurs? Mémoires du Livre/Studies in Book Culture, 1(2). http://dx.doi.org/10.7202/044212ar.

Cheng, S. W., Kuo, Chih-Wei, \& Kuo, Chih-Hua. (2012). Research article titles in applied linguistics. Journal of Academic Language \& Learning, 6(1), A1-A14.

Ciapuscio, G. (2005). Las metáforas en la creación y recontextualización de la ciencia. Signo \& Seña, 14, 183211.

Cortiñas, S. (2008). Las metáforas del ADN: Una revisión de los procesos divulgativos. Journal of Science Communication, 1(7), 1-9.

Crismore, A., \& Farnsworth, R. (1990). Metadiscourse in popular and professional science discourse. In Walter Nash (Ed.), The Writing Scholar: Studies in Academic Discourse, 118-136. Newbury Park (CA): Sage.

Day, R. A. (1998). How to Write and Publish a Scientific Paper. 5th edition. Cambridge: Cambridge University Press.

Dupriez, B. (1991). A Dictionary of Literary Devices (Gradus A-Z). Toronto: University of Toronto Press.

Fahnestock, J. (2004). Preserving the figure: Consistency in the presentation of scientific arguments. Written Communication, 21, 6-31. http://dx.doi.org/10.1177/0741088303261034.

Fischer, B. A., \& Zigmond. M. J. (2004). Components of a research article. Retrieved from http://www2.yk.psu.edu/sites/bee11/files/2011/03/Components-of-a-Research Article.pdf.

Fortanet, I., Posteguillo, S., Coll, J. F., \& Palmer, J. C. (1998). Linguistic analysis of research article titles: Disciplinary variations, In Ignacio Vázquez Orta, \& Ignacio Guillén Calvé (Eds.), Perspectivas Pragmáticas en Lingüística Aplicada, 443-447. Zaragoza (Spain): Anubar.

Frankel, F. C., \& DePace, A. H. (2012). Visual strategies: A practical Guide to Graphics for Scientists and Engineers. New Haven (CT): Yale University Press.

Giannoni, D. S. (2008). Popularizing features in English journal editorials. English for Specific Purposes, 27(2), 212232. http://dx.doi.org/10.1016/j.esp.2006.12.001.

Goodman, N. W. (2000). Survey of active verbs in the titles of clinical trial reports. British Medical Journal, 320, $914-$ 915. http://dx.doi.org/10.1136/bmj.320.7239.914.

Goodman, N. W. (2011). Fashion in medicine and language: Inferences from titles and abstracts of articles listed in PubMed. The Write Stuff, 20(1), 39-42.

Goodman, R. A., Thacker, S. B., \& Siegel, P. A. (2001). What's in a title? A descriptive study of article titles in peer reviewed medical journals. Science Editor, 24(3), 75-78.

Haggan, M. (2004). Research paper titles in literature, linguistics and science: Dimensions of attractions. Journal of Pragmatics, 36, 293-317. http://dx.doi.org/10.1016/S0378-2166(03)00090-0.

Harmon, J. E. (2009). The structure of scientific titles. Journal of Technical Writing and Communication, 39(4), 455465. http://dx.doi.org/10.2190/TW.39.4.g.

Hartley, J. (2007). Planning that title: Practices and preferences for titles with colons in academic article. Library and Information Science Research, 29, 553-568. http://dx.doi.org/10.1016/j.lisr.2007.05.002. 
Hilgartner, S. (1990). The dominant view of popularization: Conceptual problems, political uses. Social Studies of Science, 20(3), 519-539. http://dx.doi.org/10.1177/030631290020003006.

Horn, L., \& Ward G. (Eds.) (2004). The Handbook of Pragmatics. 3rd edition. Oxford: Blackwell.

Huth, E. J. (1999). Writing and Publishing in Medicine. 3rd edition. Baltimore (MD): Williams and Wilkins.

Hyland, K. (2010). Constructing proximity: Relating to readers in popular and professional science. English for Academic Purposes, 9, 116-127. http://dx.doi.org/10.1016/j.jeap.2010.02.003.

Jacques, T. S., \& Sebire, N. J. (2010). The impact of article titles on citation hits: An analysis of general and specialist medical journals. Journal of the Royal Society of Medicine Short Reports, 1(2). http://dx.doi.org/10.1258/shorts.2009.100020.

Jacobi, D. (1987). Textes et Images de la Vulgarisation Scientifique. Bern: Peter Lang.

Jaime-Sisó, M. (2009). Titles or headlines? Anticipating conclusions in biomedical research article titles as a persuasive journalistic strategy to attract busy readers. Miscelánea. A Journal of English and American Studies, 39, 29-54.

Jamali, H. R., \& Nikzad, M. (2011). Article title type and its relation with the number of downloads and citations. Scientometrics, 88(2), 653-661. http://dx.doi.org/10.1007/s11192-011-0412-z.

Jay, M. (2002). That visual turn. Journal of Visual Culture, 1, 87-92. http://dx.doi.org/10.1177/147041290200100301.

Jeanneret, Y. (1994). Écrire la Science. Formes et Enjeux de la Vulgarisation, Paris: PUF.

Kalleberg, R. (2000). Universities: Complex bundle institutions and the projects of enlightenment. Comparative Social Research, 19, 219-255.

Kecskes, I., \& Horn L. (Eds.) (2007). Explorations in Pragmatics: Linguistic, Cognitive, and Intercultural Aspects. Berlin/New York: Mouton de Gruyter.

Kyvik, S. (2005). Popular science publishing and contributions to public discourse among university faculty. Science Communication, 26, 288-311. http://dx.doi.org/10.1177/1075547004273022.

Leech, G. N. (1983). Principles of Pragmatics. London: Longman.

Lévy-Leblond, J. M. (2001). Science, culture et public: Faux problèmes et vraies questions. Quaderni, 46, 95-103. http://dx.doi.org/10.3406/quad.2001.1513.

Lewison, G., \& Hartley, J. (2005). What's in a title? Number of words and the presence of colons. Scientometrics, 63(2), 341-356. http://dx.doi.org/10.1007/s11192-005-0216-0.

Luzón Marco, M. J. (2013). Public communication of science in blogs: Recontextualizing scientific discourse for a diversified audience. Written Communication, 30, 428-457. http://dx.doi.org/10.1177/0741088313493610.

Maisonneuve, H., Lorette, G., Maruani, A., \& Huguier, M. (2010). La Rédaction Médicale. Paris: Doin editeurs.

McGowan, J., \& Tugwell, P. (2005). Informative titles described article content. Journal of the Canadian Health Libraries Association, 26, 83-84. http://dx.doi.org/10.5596/c05-029.

Méndez, D. I., Alcaraz, M. Á., \& Salager-Meyer, F. (2014). Titles in English-medium Astrophysics research articles. Scientometrics, 98(3), 2331-2351. http://dx.doi.org/10.1007/s11192-013-1174-6.

Méndez, D. I., \& Alcaraz, M. Á. (2015). The use of abbreviations in English-medium Astrophysics research paper titles: A problematic issue. Advances in Language and Literary Studies, 6(3), 185-196. http://dx.doi.org/10.7575/aiac.alls.v.6n.3p.185.

Muñoz Dagua, C. (2010). El rol de la metáfora léxica en la divulgación de la ciencia. Tabula Rasa, 13, $273-292$.

Myers, G. (1990). Writing Biology. Texts in the Social Construction of Scientific Knowledge. Wisconsin: The University of Wisconsin Press.

Myers, G. (2003). Discourse studies of scientific popularization: Questioning the boundaries. Discourse Studies, 5, 265279. http://dx.doi.org/10.1177/1461445603005002006.

Orrico, E. (2012). The Memory of scientific divulgation: An information discourse. Morpheus, 14, $128-144$.

Paiva, C. E., Lima, J. P., \& Paiva, B. S. (2012). Articles with short titles describing the results are cited more often. Clinics, 67, 509-513. http://dx.doi.org/10.6061/clinics/2012(05)17.

Pedersen, O. (2009). Early Physics and Astronomy: A Historical Introduction. 4th edition. Cambridge: Cambridge University Press.

Rodríguez Estrada, F. C., \& Davis, L. S. (2015). Improving visual communication of science through the incorporation of graphic design theories and practices into science communication. Science Communication, 37(1), 140-148. http://dx.doi.org/10.1177/1075547014562914.

Rosner, J. L. (1990). Reflections on science as a product. Nature, 345, 108.

Salager-Meyer, F., Alcaraz Ariza, M.Á., \& Luzardo Briceño, M. (2013). Titling and authorship practices in medical case reports: A diachronic approach. Communication and Medicine, 10(1), 63-80. http://dx.doi.org/10.1558/cam.v10i1.63.

Sánchez Mora, A. M. (2000). La Divulgación de la Ciencia como Literatura. Mexico: UNAM. 
Soler, V. (2007). Writing titles in science: An exploratory study. English for Specific Purposes, 26(1), 90-102. http://dx.doi.org/10.1016/j.esp.2006.08.001.

Soler, V. (2011). Comparative and contrastive observations on scientific titles written in English and Spanish. English for Specific Purpose, 30, 124-137. http://dx.doi.org/10.1016/j.esp.2010.09.002.

Smith, R. (2000). Informative titles in the BMJ. British Medical Journal, 320, 915.

Swales, J. (1990). Genre Analysis: English in Academic and Research Settings. Cambridge (UK): CUP.

Villaroya, O. (2013). An experientially-based informationless communication. In Àngels Massip-Bonet, \& Albert Bastardes-Boada (Eds.), Complexity Perspectives on Language, Communication and Society, 61-73. Heidelberg, Berlin: Springer Verlag.

Wang, Y., \& Bai, Y. (2007). A corpus-based syntactic study of medical research article titles. System, 35, 388-399. http://dx.doi.org/10.1016/j.system.2007.01.005.

\section{Notes:}

${ }^{\mathrm{i}}$ For an excellent review of the literature on the subject see, for example, Jaime-Sisó (2009) and Soler (2011).

ii An acronym is a string of letters with a syllabic structure that is usually pronounced as a word and not letter-by-letter like in the case of abbreviations.

iii Nominal titles are also called "indicative" (Huth, 1999: 90; Goodman, 2000: 914) or "descriptive" (Fischer, \& Zigmond, 2004), whereas verbal title are often referred to as "informative" (Huth, 1999: 90; Goodman, 2000: 914; McGowan, \& Tugwell, 2005: 83), "declarative" (Smith, 2000: 915), "declaratory" (Goodman, Thacker, \& Siegel, 2001: 76), "conclusion titles" (Fischer, \& Zigmond, 2004: 1), "assertive sentence title" (Rosner, 1990: 108) or "full sentence title" (Jaime-Sisó, 2009: 30; Soler, 2011: 125). Other taxonomies have also been proposed (see, for example, Harmon, 2009; Jacques, \& Sebire, 2009; Jamali, \& Nikzad, 2011; or Paiva, Lima, \& Paiva, 2012, among others).

iv The "Goldilocks" concept refers to something that must fall within some limits. It is applied across many disciplines (cognitive science and psychology, economics, medicine, etc.).

${ }^{\mathrm{v}}$ In order to not extend our paper too much, no examples of the remaining figures of speech listed in Table 7 have been included although they are available upon request. 